\title{
Validação da escala clima para criatividade em sala de aula'
}

\section{Validation study of the classroom climate for creativity scale}

\author{
Denise de Souza FLEITH \\ Leandro Silva ALMEIDA ${ }^{3}$ \\ Francisco José Brito PEIXOTO 4
}

\begin{abstract}
Resumo
O objetivo deste estudo foi adaptar e validar a Escala sobre o Clima para Criatividade em Sala de Aula para alunos da 5a série do ensino fundamental. O instrumento foi aplicado em 504 alunos de escolas públicas e particulares do Distrito Federal, a fim de se avaliar o clima de sala de aula na disciplina de língua portuguesa e de matemática. Os resultados da análise fatorial confirmatória indicaram três dimensões associadas ao clima de sala de aula para criatividade: estímulo do professor à criatividade, autopercepção do aluno sobre características criativas e motivação do aluno para aprendizagem. Os valores de alfa de Cronbach oscilaram entre 0,69 e 0,88. Conclui-se que o modelo de três fatores é adequado, e, portanto, a escala pode ser utilizada para pesquisa e avaliação do quanto o clima de sala de aula favorece o desenvolvimento da criatividade.
\end{abstract}

Unitermos: Clima de sala de aula. Criatividade. Ensino fundamental. Escala.

\begin{abstract}
The purpose of this study was to adapt and validate the Classroom Climate for Creativity Scale for $5^{\text {th }}$ grade Elementary School students. The instrument was administered to 504 students, from both public and private schools in the Federal District of Brasilia, in order to assess the classroom climate in the subjects of Portuguese and Mathematics. The results of the confirmatory factor analysis showed three dimensions associated with classroom climate for creativity: teacher's stimulus to creativity, student's self-perception of creative characteristics and student's motivation for learning. The alpha coefficient values varied between 0.69 and 0.88 . It was concluded that the three-factor model is adequate and, therefore, the scale can be used for research and assessment of the extent to which classroom climate enhances creativity.
\end{abstract}

Uniterms: Classroom climate. Creativity. Elementary school level. Scale.

Os problemas enfrentados quotidianamente pela sociedade, em função de sua elevada frequência, singularidade e complexidade, requerem respostas cada vez mais inovadoras, criativas e eficazes. Nesse sentido, a criatividade, apesar das dificuldades usuais que subsis- tem na sua definição e avaliação, permanece socialmente reconhecida como uma habilidade importante que merece ser desenvolvida e investigada. A falta de consenso acerca da definição de criatividade, a característica multidimensional e dinâmica do fenômeno

$\boldsymbol{\nabla} \mathbf{\nabla} \boldsymbol{\nabla}$

1 Apoio do Conselho Nacional de Desenvolvimento Científico e Tecnológico (Processo 400303/2007-8).

2 Universidade de Brasília, Instituto de Psicologia, Departamento de Psicologia Escolar e do Desenvolvimento. Campus Darcy Ribeiro, 70910-900, Brasília, DF, Brasil. Correspondência para/Correspondence to: D.S. FLEITH. E-mail: <fleith@unb.br>.

3 Universidade do Minho, Instituto de Educação. Campus de Gualtar, Braga, Portugal.

4 Instituto Superior de Psicologia Aplicada. Lisboa, Portugal. 
e a insuficiência de fundamentação teórica em várias medidas de criatividade constituem, sem dúvida, desafios para os pesquisadores da área (Alencar, Fleith \& Bruno-Faria, 2010; Nakano \& Wechsler, 2007; Treffinger, 1987). Como resultado, observa-se uma proliferação de estudos sobre criatividade, inclusive no Brasil, especialmente nas últimas três décadas (Santos, 1995; Zanella \& Titon, 2005, Wechsler, 2001).

Tendências recentes no estudo da criatividade sinalizam que para se compreender como, porque e quando novas ideias são produzidas, é necessário considerar tanto as variáveis intrapessoais quanto as ambientais. Sternberg e Lubart (1999) ressaltam o valor do ambiente no desenvolvimento da criatividade ao expressarem que "o indivíduo precisa de um ambiente que encoraje e reconheça suas ideias criativas. $\mathrm{O}$ indivíduo pode ter todas as condições internas necessárias ao desenvolvimento do pensamento criativo, mas sem o estímulo do ambiente, sua criatividade nunca se manifestará" (p.11). Segundo Wechsler (2006a), tanto valores e crenças pessoais como os contextos social, histórico e cultural podem influenciar o tipo de comportamento ou produto reconhecido como criativo em uma dada sociedade. Cropley (2006) argumenta que criatividade é uma força com responsabilidade social bem como um caminho para a realização individual.

Essas concepções sugerem a confluência de fatores pessoais e sociais na produção criativa, o que torna difícil a avaliação por meio de testes de criatividade disponíveis, que visam, em sua maioria, medir características individuais associadas à criatividade (Alencar \& Fleith, 2003; Almeida, Prieto, Ferrando, Oliveira \& Ferrándiz, 2008; Simonton, 1988).

Em seu modelo sistêmico de criatividade, Csikszentmihalyi (1996; 1999) esclarece que o mais importante é identificar onde está a criatividade e não o que é criatividade. Nessa perspectiva, criatividade é concebida como fruto da interação de três fatores: indivíduo (bagagem genética e experiências pessoais), domínio (cultura) e campo (sistema social). Com relação ao indivíduo, características associadas à criatividade, como curiosidade, entusiasmo, motivação e background social e cultural, são elementos cruciais para o ato criativo. O domínio reporta-se ao conjunto de conhecimentos em áreas específicas de realização, assim como 308 conjunto de regras e procedimentos simbólicos esta- belecidos culturalmente e compartilhados em uma sociedade ou por várias sociedades. Por último, o campo inclui os indivíduos que atuam como "juízes", ou seja, com a função de decidir se uma nova ideia ou produto é criativo (Csikszentmihalyi, 1996). É o campo que seleciona e retém o material a ser reconhecido, preservado e incorporado ao domínio. Professores, críticos, editores de revistas e curadores de museus são exemplos de campo. Essa proposta de análise, que oferece uma visão atual, multidimensional e integradora da criatividade, aparece amplamente mencionada na literatura (Alencar \& Fleith, 2003; Feldman, Csikszentmihalyi \& Gardner, 1994; Morais, 2001; Starko, 2001), sendo também assumida nesse artigo.

A promoção da criatividade é tema recorrente na educação. As interações na família, escola e sociedade podem estimular ou inibir o desenvolvimento da criatividade de seus membros (Chagas, Aspesi \& Fleith, 2005). Um ambiente favorável ao desenvolvimento da criatividade está relacionado à qualidade da construção dessas interações e às experiências de vida dos indivíduos inseridos nesses contextos. Amabile (1996) destaca especialmente o contexto escolar ao afirmar que "de todos os fatores e ambientes sociais que podem influenciar a criatividade, a maior parte pode ser identificada de alguma forma em sala de aula" (p.203).

Nesse sentido, é importante analisar o clima de sala de aula e em que medida ele promove ou não a criatividade dos alunos. A investigação na área aponta que, nem sempre, o desenvolvimento da criatividade e do pensamento divergente é uma preocupação da escola e do professor. Para Csikszentmihalyi (2006), as escolas ensinam a responder, mas não a questionar. Ademais, os conteúdos são abordados de forma isolada, dificultando a integração de diversas disciplinas. $\mathrm{O}$ estudo conduzido por Alencar e Fleith (2004), sobre a percepção de professores e alunos em relação à ocorrência de práticas que contribuem para o desenvolvimento da criatividade em universidades, revelou que os professores, quando comparados aos seus alunos, têm uma percepção mais favorável das práticas que contribuem para o desenvolvimento do potencial criativo. Resultados similares foram obtidos por Silva (2000), ao contrastar opiniões de alunos e professores do ensino médio sobre as características criativas dos professores e tipo de aulas ministradas. Os professores se descreviam 
como dinâmicos e motivadores, ao passo que seus alunos os percebiam como monótonos. Na pesquisa de Barreto e Martínez (2007), professores de pós-graduação reconheceram a importância da criatividade no contexto educacional e confirmaram a possibilidade de implementação de uma prática pedagógica e orientação acadêmica pautadas na criatividade.

Diante do exposto, é importante conhecer e avaliar como os ambientes de ensino e de aprendizagem na sala de aula podem favorecer ou prejudicar o desenvolvimento da criatividade dos alunos. Além disso, são poucos os instrumentos de avaliação da criatividade disponíveis no Brasil (Alencar et al., 2010; Bruno-Faria \& Alencar, 1998; Crespo, 2004; Wechsler, 2006a, 2006b). Nesse sentido, desenvolveu-se o presente estudo com vistas a examinar a validade dos resultados de uma escala sobre o clima de sala de aula para criatividade, elaborada originalmente para alunos da $3^{a}$ e $4^{a}$ séries do ensino fundamental (Fleith \& Alencar, 2005). Recorrendo dessa vezà análise fatorial confirmatória, investigou-se a organização dimensional dessa escala junto a alunos da $5^{a}$ série do ensino fundamental.

\section{Método}

\section{Participantes}

Participaram desse estudo 504 alunos de 5 a série do ensino fundamental de cinco escolas públicas e quatro escolas particulares do Distrito Federal, sendo $224(44,4 \%)$ do sexo masculino e 279 (55,4\%) do feminino (um aluno não respondeu a essa questão). Desses alunos, 298 (59,1\%) frequentavam a escola pública, ao passo que 206 (40,9\%) estavam matriculados em instituições particulares de ensino. A idade média dos participantes era de 11,5 anos (Desvio-Padrão - DP=0,81). Utilizou-se uma amostra de conveniência nesse estudo, dada a dificuldade de seleção randômica da amostra, uma vez que a participação dos sujeitos no projeto foi voluntária.

\section{Instrumentos}

Utilizou-se a Escala sobre Clima para a Criatividade em Sala de Aula, elaborada por Fleith e Alencar (2005). Trata-se de um instrumento composto de 26 itens; a resposta dos alunos é dada em uma escala do tipo likert de 5 pontos, que vai de 1 (nunca) até 5 (sempre). Essa escala foi utilizada inicialmente com crianças da $3^{a}$ e $4^{a}$ série, tendo a análise fatorial exploratória identificado cinco fatores (31\% da variância explicada): suporte da professora à expressão de ideias do aluno (cinco itens; alfa de Cronbach=0,73); autopercepção do aluno com relação à criatividade (quatro itens; alfa de Cronbach=0,65); interesse do aluno pela aprendizagem (seis itens; alfa de Cronbach=0,66); autonomia do aluno (quatro itens; alfa de Cronbach=0,55); e estímulo da professora à produção de ideias do aluno (três itens; alfa de Cronbach=0,58). Esses índices de consistência interna considerados abaixo do ideal, para além da existência de quatro itens que não foram alocados em nenhum fator, justificam a realização de novos estudos de validade da escala.

\section{Procedimentos}

Após contato com escolas com vistas a explicar o objetivo do projeto e descrever as atividades a serem desenvolvidas com os alunos, e obtida a autorização, o projeto foi encaminhado e aprovado pelo Comitê de Ética em Pesquisa da Faculdade de Ciências da Saúde da Universidade de Brasília, registro no 077/2007, em 23/ 08/2007. A escala foi então aplicada coletivamente a alunos de 5 a série do ensino fundamental por graduandos do curso de psicologia - bolsistas de iniciação científica e alunos matriculados em pesquisa -, e respondida duas vezes: uma para avaliação do clima de sala de aula para criatividade na disciplina de língua portuguesa e outra para avaliação na disciplina de matemática. As análises estatísticas foram conduzidas com a versão 17.0 do SPSS e programa AMOS associado. Para a validação da dimensionalidade da escala, por meio da análise fatorial confirmatória, foram considerados os índices de ajustamento Goodness-of-Fit Index (GFI), Adjusted Goodness-of-Fit Index (AGFI), Comparative Fit Index (CFI), Tucker-Lewis Index (TLI), Qui-Quadrado/graus de liberdade (CMIN/DF) e o Root Mean Squared Error of Approximation (RMSEA), seguindo orientações de estudiosos da área (Kline, 1998; MacCallum \& Austin, 2000).

\section{Resultados}

Uma análise fatorial exploratória foi inicialmente conduzida, observando-se uma distribuição dos itens 
por três fatores com valor próprio superior à unidade, havendo relativa homogeneidade de resultados para as disciplinas de língua portuguesa e de matemática. A solução de 3-fatores explicou 36,46\% da variância

Nas Tabelas 1, 2 e 3 são apresentados os itens pelas três dimensões, que, teoricamente, caracterizam o clima de sala de aula para criatividade na percepção dos alunos: campo - representado nesse estudo pelo professor; indivíduo - representado pelo aluno; domínio - relacionado ao conhecimento. Nessa descrição, são relatados a média e o desvio-padrão e a correlação do item com o total da subescala (corrigido). Essa análise é feita separadamente para língua portuguesa e mate- mática, considerando os itens para cada uma das três subescalas ou dimensões constituintes da escala.

Os resultados das Tabelas 1, 2 e 3 apontam para uma boa dispersão dos resultados dos alunos item a item, seja na disciplina de língua portuguesa seja na de matemática, situando-se a média dos itens em torno de 3 (valor intermédio na escala de 1 a 5 pontos) e o desvio-padrão sempre um pouco acima da unidade. Por sua vez, o índice de correlação corrigido entre o item e o total da respectiva subescala foi sempre moderado ou elevado (sempre superior a 0,30). Apenas o item 21 - Eu sou incentivado a chutar um palpite quando eu não sei a resposta para uma questão - apre-

Tabela 1. Resultados dos Itens da primeira dimensão nas disciplinas de língua portuguesa e de matemática.

\begin{tabular}{|c|c|c|c|c|c|c|c|}
\hline & & \multicolumn{6}{|c|}{ Disciplinas } \\
\hline \multirow{2}{*}{\multicolumn{2}{|c|}{ Itens da Dimensão 1}} & \multicolumn{3}{|c|}{ Língua portuguesa } & \multicolumn{3}{|c|}{ Matemática } \\
\hline & & M & DP & ritc & M & $\mathrm{DP}$ & ritc \\
\hline 1 & O professor ouve as minhas ideias & 3,03 & 1,38 & 0,70 & 3,05 & 1,43 & 0,69 \\
\hline 2 & Eu tenho oportunidade de participar de várias atividades & 3,60 & 1,22 & 0,55 & 3,67 & 1,30 & 0,64 \\
\hline 3 & Minhas ideias são bem-vindas & 3,11 & 3,45 & 0,70 & 3,11 & 1,39 & 0,73 \\
\hline 5 & O professor me incentiva a mostrar meu trabalho para outros alunos & 2,74 & 1,48 & 0,63 & 2,53 & 1,44 & 0,63 \\
\hline 7 & $\begin{array}{l}\text { O professor me dá tempo suficiente para pensar sobre uma história que tenho } \\
\text { que escrever }\end{array}$ & 3,34 & 1,48 & 0,60 & 2,98 & 1,50 & 0,63 \\
\hline 9 & Os trabalhos que faço são divertidos & 3,31 & 1,38 & 0,52 & 3,22 & 1,42 & 0,59 \\
\hline 12 & O professor se importa com o que eu tenho a dizer & 3,17 & 1,51 & 0,72 & 3,26 & 1,47 & 0,69 \\
\hline 14 & Eu sou estimulado a pensar em novas ideias & 3,24 & 1,29 & 0,59 & 3,23 & 1,37 & 0,62 \\
\hline 16 & Eu posso escolher o que eu quero fazer & 2,22 & 1,30 & 0,41 & 2,16 & 1,36 & 0,46 \\
\hline 17 & Eu sou incentivado a pensar em muitas ideias & 3,33 & 1,34 & 0,60 & 3,15 & 1,33 & 0,66 \\
\hline 21 & $\begin{array}{l}\text { Eu sou incentivado a chutar um palpite quando eu não sei a resposta para uma } \\
\text { questão }\end{array}$ & 3,10 & 1,47 & 0,16 & 2,86 & 1,49 & 0,20 \\
\hline
\end{tabular}

M: média; DP: desvio-padrão; ritc: correlação do item com o total da subescala.

Tabela 2. Resultados dos Itens da segunda dimensão nas disciplinas de língua portuguesa e de matemática.

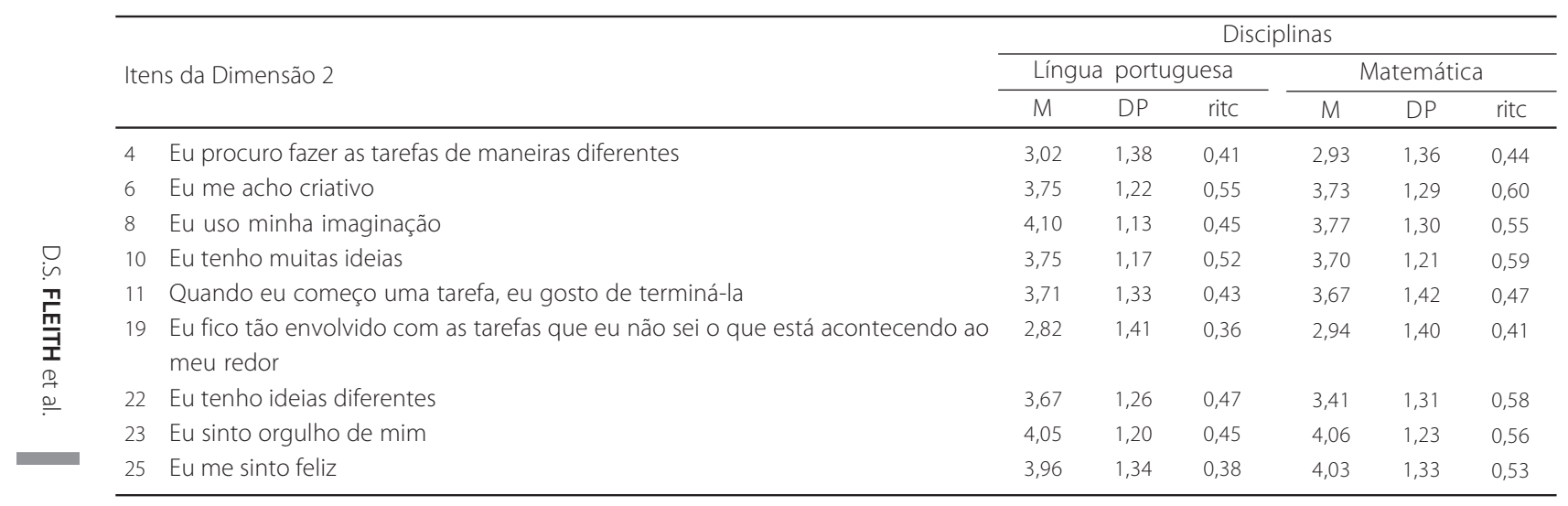

310 M: média; DP: desvio-padrão; ritc: correlação do item com o total da subescala. 
Tabela 3. Resultados dos Itens da terceira dimensão nas disciplinas de língua portuguesa e de matemática.

\begin{tabular}{|c|c|c|c|c|c|c|c|}
\hline \multirow{3}{*}{\multicolumn{2}{|c|}{ Itens da Dimensão 3}} & \multicolumn{6}{|c|}{ Disciplinas } \\
\hline & & \multicolumn{3}{|c|}{ Língua portuguesa } & \multicolumn{3}{|c|}{ Matemática } \\
\hline & & \multirow{2}{*}{$\begin{array}{l}M \\
3,30\end{array}$} & \multirow{2}{*}{$\frac{\mathrm{DP}}{1,44}$} & \multirow{2}{*}{$\begin{array}{l}\text { ritc } \\
0,49\end{array}$} & \multirow{2}{*}{$\frac{M}{3,60}$} & \multirow{2}{*}{$\frac{\mathrm{DP}}{1,44}$} & \multirow{2}{*}{$\begin{array}{l}\text { ritc } \\
0,54\end{array}$} \\
\hline 13 & Eu gosto da matéria ensinada & & & & & & \\
\hline 15 & Eu aprendo coisas que realmente me interessam & 3,65 & 1,27 & 0,51 & 3,70 & 1,34 & 0,57 \\
\hline 18 & Eu aprendo muitas coisas & 3,97 & 1,14 & 0,32 & 4,14 & 1,12 & 0,39 \\
\hline 20 & Eu pesquiso em computadores quando eu quero saber mais sobre um assunto & 2,89 & 1,50 & 0,42 & 2,55 & 1,53 & 0,37 \\
\hline 24 & $\begin{array}{l}\text { Eu pesquiso em livros, revistas ou internet quando eu quero saber mais sobre um } \\
\text { assunto }\end{array}$ & 3,18 & 1,48 & 0,48 & 2,87 & 1,52 & 0,39 \\
\hline 26 & Eu entendo a matéria ensinada & 3,73 & 1,21 & 0,31 & 3,85 & 1,23 & 0,51 \\
\hline
\end{tabular}

M: média; DP: desvio-padrão; ritc: correlação do item com o total da subescala.

senta índices baixos, especialmente para língua portuguesa. Os índices de consistência dos itens (alfa de Cronbach) variaram de 0,75 a 0,88 para língua portuguesa e de 0,80 a 0,90 para matemática.

Com base nos resultados da análise fatorial exploratória, avançou-se para a confirmação da dimensionalidade da escala considerando três fatores. $\mathrm{Na}$ Figura 1, são apresentados os resultados da análise fatorial confirmatória para a disciplina de língua portuguesa, incluindo apenas os itens que viabilizam o ajuste do modelo. Alguns itens foram eliminados, pois em certos casos estavam alocados em mais de um fator latente e, em outros, o ajuste do modelo exigia a correlação dos erros de diferentes fatores, situação não desejável. Optou-se pela eliminação de cinco itens que não favoreciam uma leitura simples e tridimensional da escala (item 9 - Os trabalhos que faço são divertidos; item 17 - Eu sou incentivado a pensar em muitas ideias; item 20 - Eu pesquiso em computadores quando eu quero saber mais sobre um assunto; item 21 - Eu sou incentivado a chutar um palpite quando eu não sei a resposta para uma questão; item 24 - Eu pesquiso em livros, revistas ou Internet quando eu quero saber mais sobre um assunto).

Na Figura 2, são apresentados os resultados da análise fatorial confirmatória para a disciplina de matemática, partindo da replicação do modelo ajustado obtido para a de língua portuguesa.

Os índices obtidos, considerando os 21 itens da escala retidos, sugerem uma boa adequação do modelo na base dos índices estatísticos obtidos tanto na disciplina de língua portuguesa [CMIN/DF=2,54; GFI=0,92; AGFI $=0,90 ; T L I=0,91 ;$ RMSEA $=0,06]$ quanto na de mate- mática [CMIN/DF=2,33; GFI=0,92; AGFI=0,90; TLI=0,93; RMSEA $=0,05]$. Assim, diante do modelo testado, é possível defender três dimensões associadas ao clima de sala de aula para criatividade: dimensão 1 - estímulo do professor à criatividade (8 itens) -, dimensão 2 - autopercepção do aluno sobre características criativas (6 itens) - e dimensão 3 - motivação do aluno para aprendizagem (7 itens). No que diz respeito à disciplina de língua portuguesa, a média, o desvio-padrão e o índice

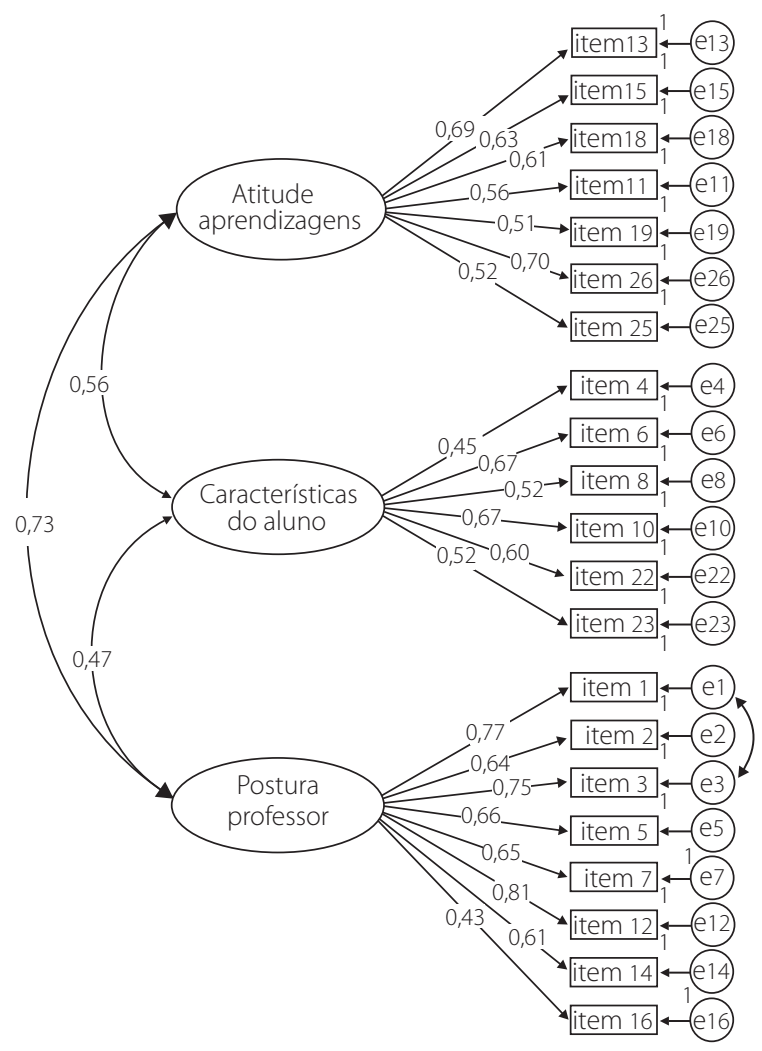

Figura 1. Resultados da análise fatorial confirmatória para o clima de sala de aula na disciplina de língua portuguesa. 
de consistência interna (alfa de Cronbach) da dimensão 1 foram respectivamente 3,07, 0,97 e 0,86; para a dimensão 2 foram 3,72, 0,81 e 0,76; e, para a dimensão 3, 3,59, 0,88 e 0,69. Na disciplina de matemática, a média, o desvio-padrão e o índice de consistência interna (alfa de (ronbach) da dimensão 1 foram, respectivamente, 3,70, 0,92 e 0,88; para a dimensão 2 foram 3,00, 1,03 e 0,82; e, para a dimensão 3, 3,60, 0,90 e 0,72.

Os resultados obtidos sugerem uma média por dimensão acima do ponto intermédio da escala Likert de 5 pontos, sugerindo uma avaliação positiva por parte do aluno em relação aos seus professores no estímulo à criatividade (na disciplina de matemática), na sua autopercepção criativa (na disciplina de português) e na sua motivação para a aprendizagem (nas duas disciplinas). Porém, os índices de consistência interna dos itens são claramente positivos em termos psicométricos para as duas primeiras dimensões, havendo uma diminuição do índice de Cronbach para a dimensão "motivação do aluno para a aprendizagem", ainda que dentro dos limiares exigidos.

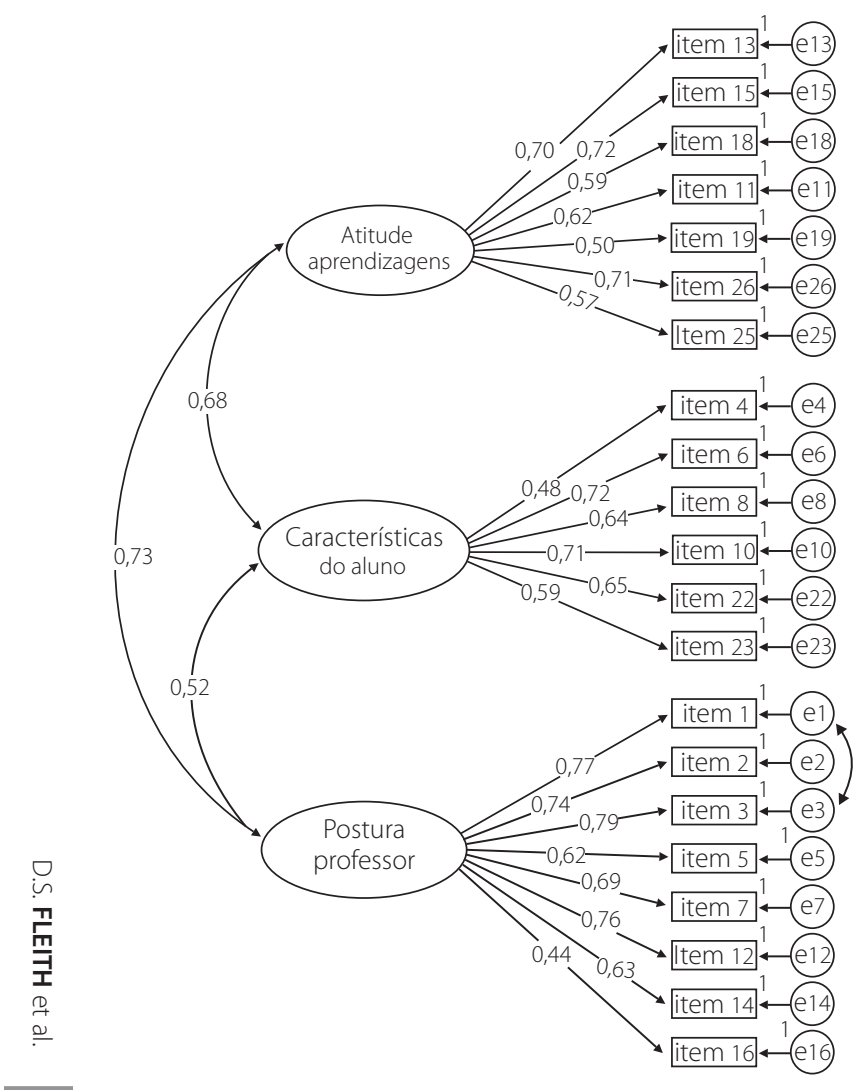

Figura 2. Resultados da análise fatorial confirmatória para o clima de sala de aula na disciplina de matemática.

\section{Discussão}

Análises preliminares da Escala sobre Clima para Criatividade em Sala de Aula apontaram alguma instabilidade na sua estrutura interna. Conforme observado no estudo original com crianças de $3^{a}$ e $4^{a}$ série do ensino fundamental, foram identificados cinco fatores - 4 dos 26 itens não foram associados a nenhum fator -, alguns deles com baixa consistência interna (índices alfa de fidedignidade inferiores a 0,70 em algumas dimensões) (Fleith \& Alencar, 2005). Em uma nova análise fatorial com alunos da $5^{\text {a }}$ série, apenas três fatores foram identificados. Foi observado, no entanto, um aumento nos índices de fidedignidade. Esses resultados sugerem ser difícil para alunos mais jovens compreender os itens do instrumento e as instruções de resposta. Conforme explica Runco (1993), crianças no início do processo de escolarização têm dificuldade em reconhecer ideias e situações criativas, embora sejam capazes de identificar ideias diferentes. Nesse sentido, deve-se evitar a aplicação da escala em alunos de séries iniciais do ensino fundamental (1 ${ }^{a} 2^{\text {a }}$ e $3^{\mathrm{a}}$ séries).

Tomando os resultados da análise fatorial confirmatória, é importante assinalar a boa adequação do modelo de três fatores para as disciplinas de língua portuguesa e de matemática. Destaca-se, ainda, que para os três fatores, os mesmos itens são referenciados para as duas disciplinas. Na análise, tanto para língua portuguesa quanto para matemática, 5 dos 26 itens foram descartados por não serem "puros" de um dos três fatores ou então por serem muito específicos. Uma provável explicação é que o conteúdo desses itens não ficou claro para os alunos ou que as situações apresentadas nos itens poderão estar bastante distantes da realidade vivida em sala de aula.

Diante dos resultados obtidos, concluímos que a Escala sobre Clima para Criatividade em Sala de Aula, a ser utilizada com alunos de $5^{\text {a }}$ série do ensino fundamental, avalia três dimensões: estímulo do professor à criatividade, autopercepção do aluno sobre características criativas, e motivação do aluno para aprendizagem. Esses achados fornecem evidências empíricas e confirmam o modelo teórico sistêmico de criatividade (Csikszentmihalyi, 1996; 1999), adotado no estudo, no qual as dimensões indivíduo (aluno), campo (professor) e domínio (interesse pela aprendizagem) são contem- 
pladas. Conclui-se, ainda, que esse instrumento construído e validado está em consonância com as concepções atuais de criatividade que defendem ser a manifestação criativa fruto da interação de fatores individuais e ambientais (Amabile, 1996; Feldman et al., 1994; Sternberg \& Lubart, 1999). A criatividade, assim, não se resume à inspiração, nem é sinônimo de dom inato (Alencar \& Fleith, 2003). Ao contrário, é resultado de preparação, de aprendizagem, de oportunidades e experiências ricas e diversificadas.

Renzulli (1992) associa a promoção de uma aprendizagem produtiva e criativa à inter-relação de três dimensões do contexto escolar - professor, aprendiz e currículo -, aspectos também considerados nos itens da Escala sobre Clima para Criatividade em Sala de Aula.

\section{Considerações Finais}

Como sugestão para futuros estudos a serem conduzidos no Brasil e em outros países, como Portugal, vale verificar o status da terceira dimensão do modelo motivação do aluno para a aprendizagem -, pois foi a que estava menos associada a esse construto (aliás, foi também a que obteve o mais baixo coeficiente de consistência interna dos respectivos itens). Nessa linha, é necessário avançar com novas investigações, reavaliando a $3^{a}$ dimensão e testando a hipótese de três fatores em alunos de diferentes faixas etárias. A expectativa é que, progressivamente, a escala seja aprimorada e venha a ser utilizada em estudos sobre o clima estimulador ou inibidor da criatividade em sala de aula no ensino fundamental.

\section{Referências}

Alencar, E. M. L. S., \& Fleith, D. S. (2003). Criatividade: múltiplas perspectivas (3a ed.). Brasília: EdUnB.

Alencar, E. M. L. S., Fleith, D. S., \& Bruno-Faria, M. F. (2010). A medida da criatividade: possibilidades e desafios. In E. M. L. S. Alencar, M. F. Bruno-Faria \& D. S. Fleith (Orgs.), Medidas de criatividade: teoria e prática (pp.11-34). Porto Alegre: Artmed.

Almeida, L. S., Prieto, L. P., Ferrando, M., Oliveira, E., \& Ferrándiz, C. (2008). Torrance Test of Creative Thinking: the question of its construct validity. Thinking Skills and Creativity, 3 (3), 53-58.

Amabile, T. A. (1996). Creativity in context. Boulder, CO: Westview Press.
Alencar, E. M. L. S., \& Fleith, D. S. (2003). Creativity in university courses: perceptions of professors and students. Gifted and Talented International, 19 (1), 24-28.

Barreto, M. O., \& Martínez, A. M. (2007). Possibilidades criativas de professores em cursos de pós-graduação stricto sensu. Estudos de Psicologia (Campinas), 24 (4), 463-473. doi: 10.1590/S0103-166X200700040006.

Bruno-Faria, M. F., \& Alencar, E. M. L. S. (1998). Indicadores de clima para criatividade (ICC): um instrumento de medida da percepção de estímulos e barreiras à criatividade no ambiente de trabalho. Revista de Administração, 33 (4), 86-91.

Chagas, J. F., Aspesi, C. C., \& Fleith, D. S. (2005). A relação entre criatividade e desenvolvimento: uma visão sistêmica. In M. A. Dessen \& A. Costa Jr. (Eds.), A ciência do desenvolvimento: tendências atuais e perspectivas futuras (pp.210-228). Porto Alegre: Artmed.

Crespo, M. L. F. (2004). Construção de uma medida de clima criativo em organizações. Estudos de Psicologia (Campinas), 21 (2), 91-99. doi: 10.1590/S0103-166X2004000200007.

Cropley, A. J. (2006). Creativity: a social approach. Roeper Review, 28 (3), 125-130.

Csikszentmihalyi, M. (1996). Creativity. New York: Harper Collins.

Csikszentmihalyi, M. (1999). Implications of a systems perspective for the study of creativity. In R. J. Sternberg (Org.), Handbook of creativity (pp.313-335). New York: Cambridge University Press.

Csikszentmihalyi, C. (2006). Developing creativity. In N. Jackson, M. Oliver, M. Shaw \& J. Wisdom (Orgs.), Developing creativity in higher education (pp.xviii-xx). London: Routledge.

Feldman, D. H., Csikszentmihalyi, M., \& Gardner, H. (1994). A framework for the study of creativity. In D. H. Feldman, M. Csikszentmihalyi \& H. Gardner (Orgs.), Changing the world: a framework for the study of creativity (pp.1-45). Westport, CT: Praeger.

Fleith, D. S., \& Alencar, E. M. L. S. (2005). Escala sobre o clima para criatividade em sala de aula. Psicologia: Teoria e Pesquisa, 21, 85-91.

Kline, R. B. (1998). Principles and practice of structural equation modeling. New York: Guilford Press.

MacCallum, R. C., \& Austin, J. T. (2000). Applications of structural equation modeling in psychological research. Annual Review of Psychology, 51 (1), 201-226.

Morais, M. F. (2001). Definição e avaliação da criatividade. Braga: Universidade do Minho.

Nakano, T., \& Wechsler, S. M. (2007). Criatividade: características da produção brasileira. Avaliação Psicológica, 6 (2), 261-273.

Renzulli, J. S. (1992). A general theory for the development of creative productivity through the pursuit of ideal acts of learning. Gifted Child Quarterly, 36, 17-182.

Runco, M. A. (1993). Cognitive and psychometric issues in creativity research. In S. G. Isaksen, M. C. Murdock, R. L. Firestien \& D. J. Treffinger (Orgs.), Understanding and 
recognizing creativity: the emergence of a discipline (pp.331-368). Norwood, NJ: Ablex.

Santos, A. T. (1995). Estudo da criatividade no Brasil:análise das teses/dissertações em psicologia e educação (1970/1993). Dissertação de mestrado não-publicada, Faculdade de Educação, Universidade Estadual de Campinas.

Silva, P. A. N. (2000). Avaliação do perfil da criatividade do professor no ensino médio. Dissertação de mestrado não-publicada, Programa de Pós-graduação em Psicologia, Pontifícia Universidade Católica de Campinas.

Simonton, D. K. (1988). Scientific genius. Cambridge: Cambridge University Press.

Starko, A. (2001). Creativity in the classroom. Mahwah, NJ: Lawrence Erlbaum Associates.

Sternberg, R. J., \& Lubart, T. I. (1999). The concept of creativity: prospects and paradigms. In R. J. Sternberg (Org.), Handbook of creativity (pp.3-15). New York: Cambridge University Press.
Treffinger, D. J. (1987). Research on creativity assessment. In S. G. Isaksen (Org.), Frontiers of creativity research: beyond the basics (pp.103-119). Buffalo, NY: Bearly.

Wechsler, S. M. (2001). Criatividade na cultura brasileira: uma década de estudos. Teoria, Investigação e Prática, 6 (1), 215-226.

Wechsler, S. M. (2006a). Validity of the Torrance tests of creative thinking to the Brazilian culture. Creativity Research Journal, 18 (1), 15-25.

Wechsler, S. M. (2006b). Estilos de pensar e criar. Campinas: PUC-Campinas.

Zanella, A. V., \& Titon, A. P. (2005). Análise da produção científica sobre criatividade em programas brasileiros de pós-graduação em psicologia (1994-2001). Psicologia em Estudo, 10 (2), 305-316.

Recebido em: 13/1/2010

Versão final reapresentada em: 25/10/2010

Aprovado em: 17/11/2010 